\title{
Winkelstabile Implantate an der unteren Extremität: Das LIS-System zur Versorgung distaler Femurfrakturen und proximaler Tibiafrakturen
}

\author{
Max Josef Kääb, Boris Conrad, Michael Schütz, Norbert P. Haas
}

\section{Zusammenfassung}

Distale Femurfrakturen und proximale Tibiafrakturen sind komplexe Verletzungen, die einen hohen Anspruch an die chirurgische Versorgung stellen. Um die Weichteiltraumatisierung durch die operative Versorgung zu minimieren, wurde das LISS (Less Invasive Stabilization System) entwickelt. Die Vorteile liegen in der winkelstabilen Verankerung, der Schonung der Knochenhautdurchblutung und der Möglichkeit eines minimalinvasiven
Vorgehens. Eigene Erfahrungen zeigen, dass bei der Verwendung des LISS niedrige Komplikationsraten bezüglich Infektion, Pseudarthrose, Achsfehlstellungen und Implantatversagen erreicht werden können. Ziel dieses Artikels ist es, einen Überblick über das LISS zur Versorgung von distalen Femur- und proximalen Tibiafrakturen zu geben. Es werden die Indikationen, die klinische Anwendung, Fallbeispiele und die eigenen Ergebnisse vorgestellt.

\section{Einführung}

\section{Das biomechanische Konzept}

Basierend auf den Resultaten der „biologischen“ Plattenosteosynthese und der Verriegelungsmarknagelung entstand 1989 das Konzept eines minimalinvasiven Systems mit Winkelstabilität [5]. Das bekannte Konzept der Winkelstabilität wurde im Hinblick auf die Klinik so modifiziert, dass die perkutane Implantation möglich war. Das Einschieben einer Platte über einen kleinen Zugang war schon mit der LC-DCP untersucht worden und wurde nun mit anatomischen Studien am LISS komplettiert, um Weichteilverletzungen bei einem ,blinden“ Einsetzen auszuschließen [5]. Die klinische Anwendung des LISS begann 1996 und bald konnte in ersten Studien über Resultate [39] berichtet werden. Die Einführung des LISS für die proximale Tibia folgte im Jahr 1998.

OP-JOURNAL 2004; 20: 28-31

(c) Georg Thieme Verlag KG Stuttgart · New York
Mit der Entwicklung des LISS wurden mehrere Ziele verfolgt. Da bekannt war, dass die Beeinträchtigung der Durchblutung des Periosts durch direkten Implantatkontakt zu verminderter Durchblutung, Knochennekrosen und damit zu verzögerter Frakturheilung, Infektion und Pseudarthrose $[6,8,15,16,20]$ führen kann, wurde bei der Entwicklung versucht, den Implantat-Knochenkontakt zu minimieren. Diese Entwicklung ging von der LC-DCP mit einer 50\% Reduzierung der Kontaktfläche gegenüber der DCP [19] über den winkelstabilen PCFix mit punktförmiger Auflagefläche bis zum LISS, das keinen Implantat-Knochenkontakt mehr benötigt.

\section{Frakturen des distalen Femur}

Zur Versorgung distaler Femurfrakturen werden extra- und intramedulläre Verfahren eingesetzt [12]. Die Ergebnisse sind mit einer nicht geringen Zahl von Infektionen, Pseudarthrosen und Fehlstellungen belastet $[18,30]$. Hauptsächliche Probleme ergeben sich aus den zusätzlichen Weichteilverletzungen und der Nähe neurovaskulärer Strukturen. Die ansetzenden Sehnen- und Bandstrukturen erschweren die anatomische Reposition. So zieht der M. gastrocnemius den
Kondylenteil des Femurs nach distal, der M. popliteus nach proximolateral, der $M$. adductor magnus inseriert an der medialen Kondyle und kann hier einen Zug nach proximal bewirken.

Wegen dieser Komplikationen wurde bis in die 70er-Jahre von einer routinemäßigen, operativen Versorgung dieser Frakturen abgeraten [30]. Erst als geeignete Implantate zur Verfügung standen und gute Ergebnisse publiziert wurden, erfolgte vermehrt eine operative Therapie [7,26,34,35]. Im Laufe der weiteren Entwicklung wurden Implantatmaterialien, Repositionstechniken, Techniken der Implantatplatzierung und die Implantate selbst verändert. Speziell bei der extramedullären Osteosynthese, bei der die Einführung der Winkelstabilität im Vordergrund stand, haben sich wichtige Veränderungen ergeben.

\section{Frakturen der proximalen Tibia}

Proximale Tibiafrakturen stellen aufgrund anatomischer und pathomorphologischer Besonderheiten eigene Ansprüche an die osteosynthetische Versorgung. So ist die sichere Verankerung intramedullärer Implantate aufgrund der anatomischen Gegebenheiten in diesem Bereich schwierig, da sich der Markraum trichterförmig nach proximal weitet. Die proximalen Tibiafrakturen entstehen entweder durch Hochrasanztraumen, durch Quetschverletzungen oder indirekte Krafteinwirkungen, die geschlossene Frakturen mit einem oft höher gradigen Weichteilschaden entstehen lassen. Der kritisch durchblutete Weichteilmantel ist oft Ausgangspunkt für Komplikationen. Die biomechanischen Besonderheiten bei der Versorgung proximaler Tibiafrakturen liegen in den Ansätzen der Muskel- und Bandstrukturen, die oftmals eine Abkippung des proximalen Fragmentes bedingen. 


\section{OP-Technik}

Das LISS ist ein anatomisch vorgeformtes Implantat (Abb.1). Das Implantat kann mithilfe eines Zielbügels zwischen Muskel und Knochen vorgeschoben werden und wird über am Handgriff montierte Trokare per Stichinzision verschraubt. Mit dem LIS-System können alle Frakturen des distalen Femurs und der proximalen Tibia versorgt werden.

\section{Distales Femur}

Die Operation wird im Regelfall in Rückenlage auf einem röntgendurchlässigen Operationstisch durchgeführt. Das verletzte Bein wird im Kniegelenk etwas gebeugt, um dem Zug auf das distale Fragment durch den M. gastrocnemius entgegenzuwirken. Bei den extraartikulären und einfach intraartikulären Frakturen erfolgt die Versorgung über einen kurzen lateralen Zugang oberhalb des distalen Femurs in Ausrichtung auf das Tub. gerdii. Bei komplexen intraartikulären Frakturen erfolgt wegen der besseren Übersichtlichkeit zur Rekonstruktion der Gelenkfläche eine laterale, parapatellare Arthrotomie.

Bei Gelenkfrakturen wird zunächst der Gelenkblock rekonstruiert und dann dieser Block an den Schaft reponiert. Nun wird das LISS unter den M. vastus lateralis nach proximal geschoben. Wesentlich ist
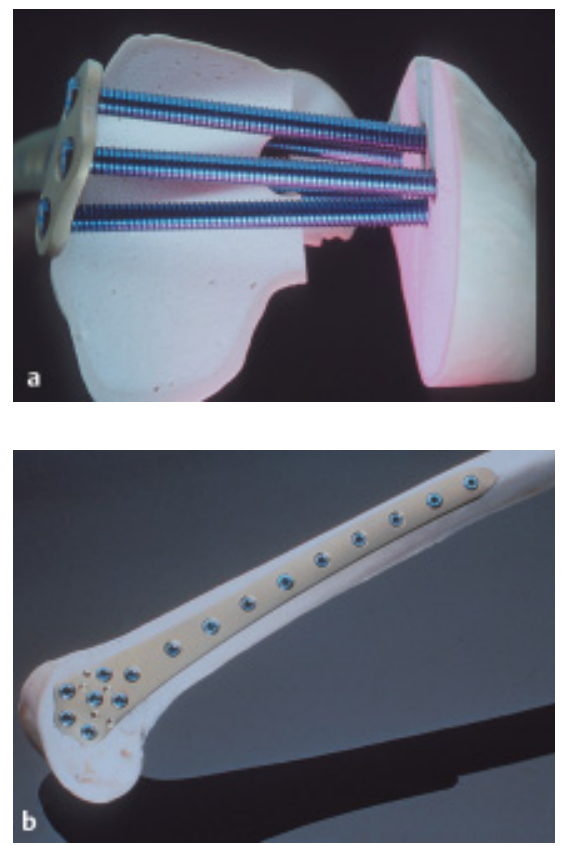

Abb.1 (a) Position der winkelstabil verankerten Schrauben in der Femurkondyle und (b) das LISS am distalen Femur. jetzt die genaue Ausrichtung am lateralen Femur, danach erfolgt die Sicherung dieser Position durch temporäre K-Drähte, die durch die Trokare eingebracht werden. Nun erfolgt die Platzierung der selbstbohrenden und selbstschneidenden Schrauben. Am Schaft erfolgt bei guter Knochenstruktur vorzugsweise die Anwendung monokortikaler Schrauben, im Gelenkbereich werden grundsätzlich lange Kondylenschrauben verwendet. Pro Fragment sollten mindes-tens 4 Schrauben zum Liegen kommen, um eine ausreichende Stabilität zu sichern.

\section{Proximale Tibia}

Ebenso ist das LISS (Abb. 2) entsprechend der Anatomie der proximalen Tibia vorgeformt und deshalb in zwei Ausführungen - für die rechte und linke Seite - verfügbar. Auch hier erlaubt ein an den Kraftträger zu montierender Handgriff das perkutane, minimal invasive Einbringen.

Mit dem LISS kann ein breites Spektrum an proximalen Tibiafrakturen abgedeckt werden. Vorteilhaft können auch intraartikuläre Frakturen mit einem weit nach distal reichenden Frakturverlauf versorgt werden.
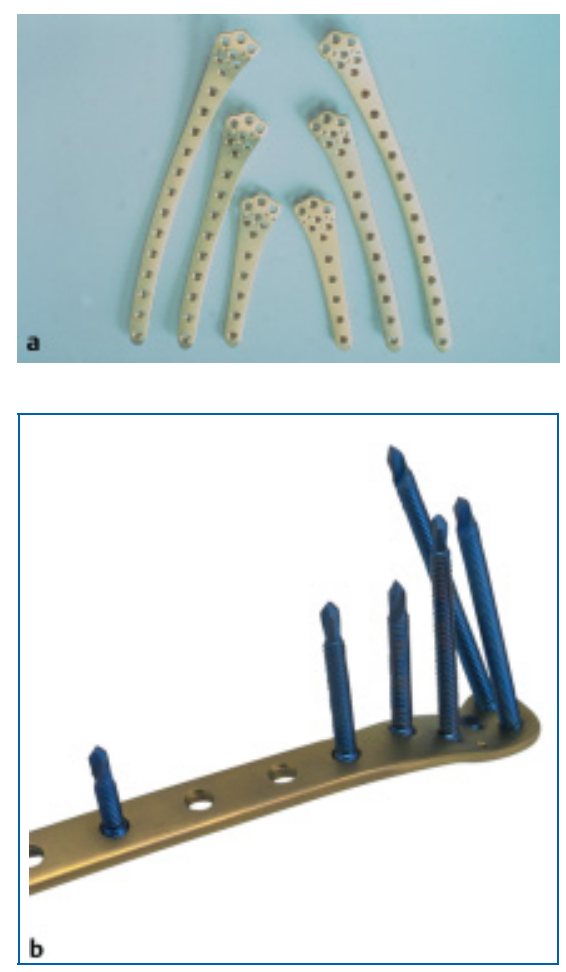

Abb. 2 (a) zeigt das anatomisch vorgeformte LISS für die proximale Tibia (PT), das in 3 verschiedenen Längen (5, 9, 13-Loch) verfügbar ist. (b) zeigt eine Detailaufnahme des proximalen Anteils der LISS PT. In Bild (c) ist der mit dem Handgriff gebildete Rahmen zu sehen und (d) illustriert die Verschraubung über die Trokarhülsen.


Bei extraartikulären Frakturen sollte zuerst die Reposition der Fraktur erfolgen, da die Repositionsmöglichkeiten über das Implantat und bei liegendem Implantat limitiert sind. Unter Umständen kann ein temporärer Fixateur externe oder ein Distraktor notwendig sein. Der operative Zugang erfolgt über eine laterale Inzision über dem Tibiakopf mit einer leichten ventralen Orientierung.

Bei intraartikulären Frakturen erfolgt zuerst die Wiederherstellung der Gelenkflächen. Ist der Gelenkblock rekonstruiert, wird die extraartikuläre Fraktur reponiert und retiniert.

\section{Ergebnisse}

\section{Distale Femurfrakturen}

Von Dezember 1996 bis November 1998 erfassten einschließlich unserer Klinik neun europäische Zentren prospektiv 112 Patienten mit 116 distalen Femurfrakturen, die mit dem LIS-System versorgt wurden [26]. Es wurden 64 weibliche und 48 männliche Patienten behandelt, das Durchschnittsalter lag bei 54 Jahren. Bei 60 Patienten lagen isolierte Frakturen und bei 19 multiple Frakturen vor. $37 \mathrm{~Pa}$ tienten waren polytraumatisiert. Die 



Abb.3 (a) Unfallbild. Die postoperativen Aufnahmen (b) 4 Wochen und (c) 6 Monate nach der Versorgung. Bild (d) zeigt die Kniebeugung der betroffenen Extremität 6 Monate nach Operation.

Frakturverteilung ergab 85 distale Femurfrakturen Typ AO 33 (76\%) und 31 Femurschaftfrakturen vom Typ AO 32 (24\%). Es traten 12 periprothetische Frakturen auf, in 6 Fällen wurde primär ein anderes Implantat zur Frakturstabilisierung eingesetzt und 11 Patienten hatten einen drittgradigen Weichteilschaden bei offener Fraktur. Die Reposition erfolgte in 73 Fällen manuell, 25-mal wurde ein Fixateur externe benutzt, in 6 Fällen wurde ein Distraktor eingesetzt und 3-mal der Frakturtisch. In 9 Fällen wurden die Methoden kombiniert. Die Nachuntersuchungsrate lag bei $93 \%$ ( $n=96$ mit 99 Frakturen).

In $91 \%$ der nachuntersuchten Fälle konnte eine sichere knöcherne Durchbauung erreicht werden. 4 Fälle zeigten keine vollständige Durchbauung und 5 Fälle waren bei Abschluss der Untersuchung noch in weiterführender Behandlung.

Es traten in 23 Fällen folgende Komplikationen auf: Sechs Fälle bedurften einer sekundären Spongiosaplastik bei verzögerter oder ausbleibender knöcherner Durchbauung. Hiervon waren alle Frakturen intraartikulär (AO 33 C3) und hatten einen zusätzlichen zweit- oder drittgradigen Weichteilschaden. Viermal kam es zu einer Implantatlockerung, zweimal zum Implantatbruch. Infektionen ließen in 7 Fällen Debridements notwendig werden. Aufgrund von starken Vernarbungen und überschießender Knochenbildung waren drei Gelenklysen notwendig. Eine Fraktur vom Typ AO 33 C2 wies nach primärer Versorgung eine zu starke Achsabweichung auf, so dass eine Umstellungsosteotomie vorgenommen wurde, die zur erfolgreichen Ausheilung führte.

\section{Proximale Tibiafrakturen}

In unserer Klinik wurden von Juli 1998 bis März 2001 insgesamt 31 Tibiafrakturen aller Schweregrade prospektiv erfasst und mit LISS versorgt (Abb. 3). Der Anteil der offenen Frakturen betrug 35\%. Die proximalen Tibiafrakturen mit der AOKlassifikation 41 summierten sich auf $68 \%$ der Fälle, $32 \%$ waren AO-42-Frakturen. $76 \%$ der Frakturen waren intraartikulär. Bei der Reposition wurde in 13 von 31 Fällen ein Fixateur externe benutzt, der in allen Fällen zur primären Stabilisierung im Rahmen von Mehrfachverletzungen appliziert worden war.

Bei den 29 Patienten, die nachuntersucht werden konnten (94\% Follow-up), wurden eine Plattenlockerung, ein Plattenbruch und eine Infektion beobachtet. Insgesamt kam es zu 2 verzögerten Frakturheilungen und es wurden 2 sekundäre Spongiosaplastiken durchgeführt. In 2 Fällen wurde eine Achsfehlstellung von $10^{\circ}$ postoperativ festgestellt. Sekundäre Repositionsverluste wurden nicht beobachtet.

\section{Schlussfolgerung}

Hauptvorteil des LISS ist die Möglichkeit des minimalinvasiven Einbringens. Die klinischen Ergebnisse zur Versorgung von Frakturen am distalen Femur und an der proximalen Tibia zeigen gute funktionelle und radiologische Ergebnisse bei relativ wenig Komplikationen wie Infektion, Pseudarthrose, Implantatversagen oder die Notwendigkeit primärer Spongiosaplastiken [11,27,28]
Ein weiterer Vorteil der LISS ist das breite Indikationsspektrum. Mit dem System können alle Frakturen des distalen Femurs therapiert werden: periprothetische Femurfrakturen, distale Femurschaftfrakturen, extraartikuläre distale Femurfrakturen und komplexe intraartikuläre Trümmerfrakturen. An der proximalen Tibia sind weit gehend alle Frakturen in das Indikationsspektrum eingeschlossen. Auch hier können intraartikuläre Frakturen mit einem weit nach distal reichenden Frakturverlauf gut versorgt werden. Besonders beim osteoporotischen Knochen ermöglicht das Fixateurinterne-Konzept eine hohe Verankerungsstabilität [29].

Das LISS hat sich am distalen Femur und an der proximalen Tibia aufgrund hoher Stabilität durch die winkelstabile Verankerung und die Möglichkeit des minimal invasiven Vorgehens bewährt.

\section{Literatur}

${ }^{1}$ Baumgaertel F, Gotzen L. Die „biologische“ Plattenosteosynthese von Mehrfragmentfrakturen des gelenknahen Femurs. Eine prospektive Studie. Unfallchirurg 1994; 97(2): $78-84$

${ }^{2}$ Bolhofner BR, Carmen B, Clifford P. The Results of Open Reduction and Internal Fixation of Distal Femur Fractures Using a Biologic (Indirect) Reduction Technique. J Orthop Trauma 1996; 10(6): 372-377

${ }^{3}$ Farouk O, Krettek C, Miclau T, Schandelmaier P, Guy P, Tscherne H. Minimally invasive plate osteosynthesis and vascularity: preliminary results of a cadaver injection study. Injury 1997; 28 Suppl 1: A7-A12

${ }^{4}$ Frank O, Sutter PM, Regazzoni P. Erste Erfahrungen mit dem Less Invasive Stabilization System (LISS) bei distalen Femurfrakturen. Swiss Surg 2000; 6 Suppl 1: 28

${ }^{5}$ Frigg R, Appenzeller A, Christensen R, Frenk A, Gilbert S, Schavan R. The development of 
the distal femur Less Invasive Stabilization System (LISS). Injury 2001; 32 Suppl 3: 24-31

${ }^{6}$ Gautier E, Perren SM. Die „Limited Contact Dynamic Compression Plate“ (LC-DCP) - Biomechanische Forschung als Grundlage des neuen Plattendesigns. Orthopäde 1992; 21(1): $11-23$

${ }^{7}$ Haas N, Schandelmaier P, Krettek C. Therapeutisches Konzept bei der distalen Femurfraktur mit Gelenkbeteiligung. Hefte Unfallheilkd 1990; 212: 179-187

${ }^{8}$ Jacobs RR, Rahn BA, Perren SM. Effects of plates on cortical bone perfusion. J Trauma 1981; 21(2): $91-95$

${ }^{9}$ Johnson EE. Combined direct and indirect reduction of comminuted four-part intraarticular T-type fractures of the distal femur. Clin Orthop 1988; (231): 154-162

${ }^{10}$ Kregor PJ, Stannard J, Cole PA, Zlowodzki M Alonso J. Prospective clinical trial of the less invasive stabilization (L.I.S.S.) for supracondylar femur fractures. J Orthop Trauma 2000; 14(2): 133-134

${ }^{11}$ Kregor PJ, Stannard J, Zlowodzki M, Cole PA, Alonso J. Distal femoral fracture fixation utilizing the Less Invasive Stabilization System (L.I.S.S.): The technique and early results. Injury 2001; 32 Suppl 3: $32-47$

${ }^{12}$ Krettek C, Schandelmaier P, Miclau T, Tscherne $\mathrm{H}$. Minimally invasive percutaneous plate osteosynthesis (MIPPO) using the DCS in proximal and distal femoral fractures. Injury 1997; 28 Suppl 1: A20 - A30

${ }^{13}$ Krettek C, Schandelmaier P, Tscherne H. Distale Femurfrakturen. Transartikuläre Rekonstruktion, perkutane Plattenosteosynthese und retrograde Nagelung [Distal femoral fractures. Transarticular reconstruction, percutaneous plate osteosynthesis and retrograde nailing]. Unfallchirurg 1996; 99(1): $2-10$

${ }^{14}$ Lagana S, Kübler N, Babst R. Erste Erfahrungen mit dem Less invasive stabilising system (LISS) bei distalen Femurfrakturen. Swiss Surg 2000; 6 Suppl 1: 27

${ }^{15}$ Matter P, Burch HB. Clinical experience with titanium implants, especially with the limited contact dynamic compression plate system. Arch Orthop Trauma Surg 1990; 109(6): $311-313$

${ }^{16}$ Melcher GA, Claudi B, Schlegel U, Perren SM, Printzen G, Munzinger J. Influence of type of medullary nail on the development of local infection. An experimental study of solid and slotted nails in rabbits. J Bone Joint Surg Br 1994; 76(6): 955-959

${ }^{17}$ Miclau T, Remiger A, Tepic S, Lindsey R, McIff T. A mechanical comparison of the dynamic compression plate, limited contact-dynamic compression plate, and point contact fixator. J Orthop Trauma 1995; 9(1): $17-22$

18 Olerud S. Operative treatment of supracondylar-condylar fractures of the femur. Technique and results in fifteen cases. J Bone Joint Surg Am 1972; 54(5): 1015-1032

19 Perren SM, Buchanan J. Basic concepts relevant to the design and development of the point contact fixator (PC-Fix). Injury 1995; 26 Suppl 2: B1 - B4

${ }^{20}$ Perren SM, Cordey J, Rahn BA, Gautier E, Schneider E. Early temporary porosis of bone induced by internal fixation implants. A reaction to necrosis, not to stress protection? Clin Orthop 1988; (232): 139-151

${ }^{21}$ Perren SM. Editorial: Minimally invasive internal fixation: history,essence and potential of a new approach, Injury, Int J Care Injured 2001; 32: S-A-1-3

${ }^{22}$ Predieri M, Gautier E, Sutter F, Tepic S, Perren SM. Vermeidung der Porose unter Osteosyntheseplatten. Acta med Austriaca 1990; 40(Suppl): $48-49$

${ }^{23}$ Sanders R, Regazzoni P, Ruedi TP. Treatment of supracondylar-intracondylar fractures of the femur using the dynamic condylar screw: J Orthop Trauma 1989; 3(3): $214-$ 222

${ }^{24}$ Schandelmaier P, Partenheimer A, Koenemann B, Grün OA, Krettek C. Distal femoral fractures and LISS stabilization. Injury 2001; 32 Suppl 3: 55-63

${ }^{25}$ Schandelmaier P, Stephan C, Krettek C, Tscherne H. Distale Femurfrakturen. Unfallchirurg 2000; 103(6): 428-436

${ }^{26}$ Schatzker J, Lambert DC. Supracondylar fractures of the femur. Clin Orthop 1979; (138): 77-83

${ }^{27}$ Schütz M, Kääb MJ, Haas N. Stabilization of proximal tibial fractures with the LIS-System: Early clinical experience in Berlin, Injury $2003 ; 34$ : S-A-30-S-A-35

${ }^{28}$ Schütz M, Müller M, Krettek C, Höntzsch D, Regazzoni P, Ganz R, Haas N. Minimally invasive fracture stabilization of distal femoral fractures with LISS: A prospective multicenter study. Injury 2001; 32: S-C-48-54

${ }^{29}$ Seebeck J, Lill C, Morlock MM, Schneider E. Implantatverankerung in osteoporotischen Knochen mittels monokortikaler Schrauben. Trauma Berufskrankh 1999; 1: 411 - 41

${ }^{30}$ Seinsheimer F. Fractures of the distal femur Clin Orthop 1980; (153): 169-179

${ }^{31}$ Tegner Y, Lysholm J. Rating systems in the evaluation of knee ligament injuries. Clin Orthop 1985; (198): 43-49

${ }^{32}$ Tepic S, Perren SM. The biomechanics of the PC-Fix internal fixator. Injury 1995; 26 Suppl 2: $5-10$

${ }^{33}$ Thielemann FW, Blersch E, Holz U. Die Plattenosteosynthese der Femurschaftfraktur unter Beachtung biologischer Gesichtspunkte. Unfallchirurg 1988; 91(9): 389-394

${ }^{34}$ Trentz O, Tscherne H, Oestern HJ. Operationstechnik und Ergebnisse bei distalen $\mathrm{Fe}$ murfrakturen. Unfallheilkunde 1977; 80(11): $441-448$

${ }^{35}$ Tscherne H, Oestern H, Trentz O. Spätergebnisse der distalen Femurfraktur und ihre besonderen Probleme. Zentralbl Chir 1977; 102(15): $897-904$

Dr. Dr. med. Max Josef Kääb

Oberarzt

Boris Conrad

Doktorand

Priv.-Doz. Dr. med. Michael Schüitz

Oberarzt

Prof. Dr. med. Norbert P. Haas

Ärztlicher Direktor

Zentrum für Muskuloskeletale

Chirurgie

Klinik für Orthopädie und Klinik

für Unfall- und Wiederherstellungs-

chirurgie

Campus Virchow-Klinikum

Augustenburger Platz 1

D-13353 Berlin 